Methods ECG electrodes and a PO sensor were attached as soon as possible after birth in healthy pre (term) infants. PO data with Signal Identification and Quality $<0.30$ were excluded. $\mathrm{HR}_{\mathrm{ECG}}$ and $\mathrm{HR}_{\mathrm{PO}}$ were compared every $30 \mathrm{~s}$ from 1-10 min.

Results 755 data pairs of 53 patients were analysed. Median (range) gestational age was 36 (27-41) weeks. Data from ECG and PO were acquired from 82 (26) and 99 (33)s after birth. Median $\mathrm{HR}_{\mathrm{PO}}$ was $<100 \mathrm{bpm}$ the first 2 min after which it rapidly increased. $\mathrm{HR}_{\mathrm{ECG}}$ remained stable at 150-160 bpm (table). Conclusion HR measured by PO is significantly lower compared to ECG, which has important implications for clinical care in the first minutes after birth.

\section{PS-104 EARLY MARKERS OF AN INCREASED CARDIOVASCULAR RISK IN FORMER PRETERM TYROLEAN PRESCHOOLERS - PRELIMINARY DATA}

${ }^{1}$ A Posod, ${ }^{1}$ I Odri Komazec, ${ }^{1} \mathrm{~K}$ Kager, ${ }^{1} \mathrm{U}$ Pupp Peglow, ${ }^{2} \mathrm{D}$ Baumgartner, ${ }^{1} \mathrm{U}$ Kiechl-Kohlendorfer. ${ }^{1}$ Pediatrics II (Neonatology), Innsbruck Medical University, Innsbruck, Austria; ${ }^{2}$ Pediatrics III (Cardiology Pulmonology Allergology and Cystic Fibrosis), Innsbruck Medical University, Innsbruck, Austria

\subsection{6/archdischild-2014-307384.399}

Background and aims Cardiovascular disease is the leading cause of death worldwide. A growing body of evidence suggests that preterm infants develop an unfavourable cardiovascular risk profile in adult life. The aim of this study was to investigate whether early laboratory and ultrasonographic markers of an increased cardiovascular risk are present in former preterm infants at a preschool age.

Methods Former preterm infants born in Tyrol between 2006 and 2008 with a gestational age $<32$ weeks were followed up at a corrected age of five to seven years. Healthy preschoolers born at term served as controls. Glucose and cholesterol levels were determined after a minimum overnight fasting period of twelve hours. Aortic intima-media thickness (aIMT) was assessed by means of high resolution ultrasound and a software quantification tool.

Results 137 children (preterm: 64, term: 73) were examined. Blood samples were obtained from 80 participants (preterm: 57, term: 23). In comparison to children born at term, former preterm infants had significantly higher HDL cholesterol, but also fasting glucose levels. Total and LDL cholesterol levels tended to be higher in the preterm group, but these findings did not reach statistical significance. aIMT measurements did not differ between groups. All parameters were independent of current BMI percentile and gender.

Conclusions In comparison to children born at term, former preterm infants have higher fasting glucose levels and show dualistic cholesterol alterations at a preschool age. The relevance of these findings with regard to future cardiovascular health will be addressed by additional studies.

\section{PS-105 LEFT VENTRICULAR MASS AND DIASTOLIC FUNCTION IN NORMOTENSIVE AND OBESE CHILDREN}

${ }^{1} \mathrm{M}$ Porcar, ${ }^{2} \mathrm{M}$ Tuzón, ${ }^{2} \mathrm{~V}$ Girbés, ${ }^{3} \mathrm{M}$ Navarro, ${ }^{3} \mathrm{~J}$ Carrasco, ${ }^{3} \mathrm{P}$ Codoñer. ${ }^{1}$ Dr. Peset University Hospital, Department of Pediatrics, Valencia, Spain; ${ }^{2}$ Dr. Peset University Hospital, Department of Cardiology, Valencia, Spain; ${ }^{3}$ Dr. Peset University Hospital, Department of Pediatrics Obstetrics and Gynecology, Valencia, Spain

10.1136/archdischild-2014-307384.400
Backgrounds and aims Several studies have stated the association between higher left ventricular mass and impaired systolic and diastolic function in obese adults. However, little is known in paediatric population. The aim of this study is to assess how early changes appear in obese and normotensive patients.

Methods Anthropometric and biochemical measurements were taken in 130 normotensive children, 88 with obesity (age 11.0 \pm 2.8 years) and 42 with normal weight (age $11.1 \pm 2.7$ years). The function and left ventricular (LV) mass was assessed according to the formula of Devereux and indexed for height ${ }^{2.7}$ to obtain left ventricular mass index (LVMI). The E/E' value, quotient between transmitral early filling wave (E) and the average of the diastolic anular velocity (E') was calculated for estimating the left ventricular filling pressure, therefore diastolic function.

Results The obese group showed higher levels of LVMI than control group $\left(43.6 \pm 14.2 \mathrm{~g} / \mathrm{m}^{2} .7\right.$ vs $36.3 \pm 13.3 \mathrm{~g} / \mathrm{m}^{2} .7, \mathrm{p}=$ $0.020)$, and higher levels of ratio $\mathrm{E} / \mathrm{E}^{\prime}$ (6.03 \pm 0.97 vs $7.24 \pm$ $1.09, \mathrm{p}<0.001)$. The areas under the curve are 0.679 for LVMi and 0.757 for $\mathrm{E} / \mathrm{E}$ '. Therefore, $\mathrm{E} / \mathrm{E}$ ' parameter is more valid than LVMi with respect the excess of BMI. The cut-off point to $\mathrm{E} / \mathrm{E}^{\prime}$ between obese and normal weight children was 6.55 .

Conclusion In order to select one of the two main cardiac alterations found, diastolic function is a more sensitive/early finding than increased LV mass in obese normotensive children.

\section{PS-106 OUTCOME OF NARROW COMPLEX ARRHYTHMIAS IN INFANTS: A SIX-YEAR RETROSPECTIVE STUDY}

${ }^{1} \mathrm{~A}$ Khushu, ${ }^{2} \mathrm{~W}$ Kelsall, ${ }^{3} \mathrm{R}$ Yates, ${ }^{2}$ Y Singh. ${ }^{1}$ School of Clinical Medicine, University of Cambridge, Cambridge, UK; ${ }^{2}$ Paediatrics, Cambridge University Hospitals NHS Foundation Trust, Cambridge, UK; ${ }^{3}$ Paediatric Cardiology, Great Ormond Street Hospital, Cambridge, UK

\subsection{6/archdischild-2014-307384.401}

Background Supraventricular tachycardia (SVT) is the most common arrhythmia in infants.

Aim To study the clinical presentation and outcome of narrow complex cardiac arrhythmias in infants

Methods All infants diagnosed with narrow complex arrhythmias in children less than 1 year of age between 2007 and 2013 at Addenbrooke's hospital were included in the study.

Results 34 infants diagnosed with narrow complex arrhythmias were identified. Eighteen (53\%) infants presented within first 7 days after birth, 8 (24\%) between 1-4 weeks and the remaining $8(24 \%)$ presented after 4 weeks of age.

Only 3 infants had antenatal diagnoses of arrhythmia. SVT was diagnosed in 27 infants (80\%). Other types of arrhythmias included atrial flutter (2) and automated atrial tachycardia (5). Eight infants had underlying significant congenital heart disease.

Fifteen infants were excluded from study as 3 died of other serious underlying conditions, 5 transferred to other hospitals and 7 did not require maintenance therapy.

Of the 19 infants diagnosed with SVT needing maintenance therapy, $6(32 \%)$ are still on maintenance therapy while in 13 $(68 \%)$ infants maintenance therapy was stopped. Ten had no recurrences after stopping maintenance while three had recurrences needing re-starting treatment.

The average duration of medical treatment was 8 months. Maintenance medications used included propranolol, flecainide and amiodarone. $63 \%$ cases had monotherapy while $37 \%$ needed multi-drug therapy.

Conclusion Narrow complex tachycardia carries good prognosis in infants without any underlying morbidities. About two thirds 